\title{
Recent CMS results on exclusive processes
}

\author{
Alexander Bylinkin* \\ The University of Kansas \\ E-mail: alexander.bylinkinegmail.com
}

\begin{abstract}
Exclusive vector meson photoproduction is studied in ultra-peripheral $\mathrm{pPb}$ collisions at $\sqrt{s_{N N}}=$ $5.02 \mathrm{TeV}$. The cross sections are measured as a function of the photon-proton centre-of-mass energy, extending the energy range explored by $\mathrm{H} 1$ and ZEUS Experiments at HERA. In addition, the differential cross sections $(d \sigma / d|t|)$, where $|t| \approx p_{T}^{2}$ is the squared transverse momentum of produced vector mesons, are measured and the slope parameters are obtained. The results are compared to previous measurements and to theoretical predictions.
\end{abstract}

XXVII International Workshop on Deep-Inelastic Scattering and Related Subjects - DIS2019

8-12 April, 2019

Torino, Italy

\footnotetext{
* Speaker.

${ }^{\dagger}$ on behalf the CMS Collanoration.
} 


\section{Introduction}

The exclusive production in proton-proton (or proton-ion) collisions, $p p(A) \rightarrow p+X+p(A)$, is characterized by the produced system $X$, where $X$ is emitted at central rapidities $y$ and the incident protons stay intact or dissociate without detection. Exclusive production in proton-proton collisions at central rapidities is usually described in terms of double pomeron exchange (DPE) processes, when the mass of the central system is not very large, or perturbatively, as in central exclusiveproduction approaches where the partonic structure of the pomeron (color singlet exchange with vacuum quantum numbers) is explicitly taken into account. In turn, proton-ion collisions allow to study high-energy photon-induced interactions with proton and/or ion beams, due to the high energy and large integrated luminosities available at the LHC. The CMS detector [1] provides a very wide range of opportunities to study both of these processes.

\section{Exclusive $\Upsilon$ photoproduction in $\mathbf{p P b}$ collisions}

Exclusive photoproduction of heavy vector mesons (Figure 1) at very high photon-proton centre-of-mass energies $\left(W_{\gamma p}\right)$ can be studied in ultraperipheral collisions (UPC) off protons (ions). Recently, CMS [2] and LHCb [3] presented their measurements of exclusive heavy $\Upsilon$ photoproduction at the LHC. The process occurs through $\gamma p$ or $\gamma P b$ interactions via the exchange of two-gluons with no net color transfer and thus, at leading order (LO), the cross-section is proportional to the square of the gluon density in the target proton or ion. It provides a valuable probe of the gluon density at a small momentum fraction $x$, which is kinematically related to $W_{\gamma p}\left(x=\left(M_{\Upsilon} / W_{\gamma p}\right)^{2}\right)$.
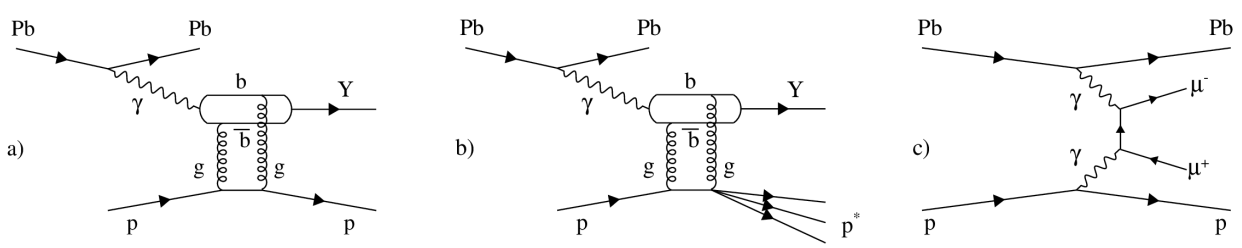

Figure 1: Diagrams representing exclusive (left) and proton-dissociative (center) $\Upsilon$ photoproduction, and exclusive dimuon QED continuum (right) in $\mathrm{pPb}$ collisions.

The exclusive photoproduction of $\Upsilon(1 S, 2 S, 3 S)$ has been measured in their dimuon decay channel in ultraperipheral collisions of protons and heavy ions $(\mathrm{pPb})$ with the CMS experiment at $\sqrt{s_{N N}}=5.02 \mathrm{TeV}$ for an integrated luminosity of $L_{\text {int }}=33 \mathrm{nb}^{-1}$.

The UPC events were selected by applying a dedicated trigger that selects at least one muon in each event and at least one to six tracks. To select the exclusive $\Upsilon(\mathrm{nS})$ events offline, two muon tracks originating from the same primary vertex in each event were used. The muons were selected with $p_{T}>3.3 \mathrm{GeV}$ and pseudorapidity $|\eta|<2.2$, in order to have high muon finding efficiency. The $p_{T}$ of the muon pair was selected between 0.1 to $1 \mathrm{GeV}$. The lower cut on the muon pair reduces the contamination from elastic QED background and the higher cut on the muon pair reduces the contamination from inelastic background (proton dissociation, inclusive $\Upsilon$, Drell-Yan). The rapidity of the muon pair is restricted to $|y|<2.2$.

These backgrounds were subtracted from data to get the exclusive signal. The background subtracted $|t|$ and $y$ distributions were used to measure the $b$ parameter and estimate the exclusive 
$\Upsilon$ photoproduction cross-section as a function of $W_{\gamma p}$, respectively. The distributions were first unfolded to the region $0.01<|t|<1(\mathrm{GeV} / c)^{2},|y|<2.2$, and muon $p_{T}^{\mu}>3.3 \mathrm{GeV}$, using the iterative Bayesian unfolding technique and its further extrapolation to zero transverse momenta by an acceptance correction factor.

The differential $d \sigma / d t$ cross section is extracted for the combined three $\Upsilon(n S)$ states as shown in Figure 2 (left), according to

$$
\frac{d \sigma_{\Upsilon}}{d|t|}=\frac{N^{\Upsilon(n S)}}{L \cdot \Delta|t|}
$$

where $|t|$ is approximated by the dimuon transverse momentum squared $p_{T}^{2}, N^{\Upsilon(n S)}$ denotes the background-subtracted, unfolded and acceptance-corrected number of signal events in each $|t|$ bin, $L$ is the integrated luminosity, and $\Delta|t|$ is the width of each $|t|$ bin. A value of $b=4.5 \pm 1.7$ (stat. $) \pm$ 0.6 (syst.) $\mathrm{GeV}^{-2}$ is extracted from the exponential fit of the measured distribution. This result is in agreement with the value $b=4.3_{-1.3}^{+2.0}($ stat. $) \mathrm{GeV}^{-2}$ measured by the ZEUS experiment [4] for the photon-proton center-of-mass energy $60<W_{\gamma p}<220 \mathrm{GeV}$. The measured value of $b$ is also consistent with the predictions based on pQCD models [5].

The differential $\Upsilon(1 S)$ photoproduction cross section $d \sigma / d y$ is corrected by the feed-down contribution to the $\Upsilon(1 S)$ events and the branching ratio for muonic $\Upsilon(1 S)$ decays $B R=(2.48 \pm$ $0.05)$. The exclusive $\Upsilon(1 S)$ photoproduction cross section as a function of $W_{\gamma p}$ as shown in Figure 2 (right), is obtained by using,

$$
\sigma_{\gamma p \rightarrow \Upsilon(1 S) p}\left(W_{\gamma p}^{2}\right)=\frac{1}{\Phi} \frac{d \sigma_{\Upsilon(1 S)}}{d y}
$$

where $\Phi$ is the photon flux evaluated at the mean of the rapidity bin, estimated from STARLIGHT. The CMS data are plotted together with the previous measurements from H1 [6], ZEUS [7] and LHCb [3] data. It is also compared with different theoretical predictions of the JMRT model [5], factorized IPsat model [8], IIM [9, 10] and bCGC model [11]. As $\sigma\left(W_{\gamma p}\right)$ is proportional to the square of the gluon PDF of the proton and the gluon distribution at low Bjorken $x$ is well described by a power law, the cross section will also follow a power law. Any deviation from such trend would indicate a different behavior of the gluon density function. We fit a power-law $A \cdot(W / 400)^{\delta}$ with CMS data alone, which gives $\delta=0.96 \pm 0.43$ and $A=655 \pm 196$ and is shown by the black solid line. The extracted $\delta$ value is comparable to the value $\delta=1.2 \pm 0.8$, obtained by ZEUS [7].

\section{Exclusive $\rho(770)^{0}$ photoproduction in pPb collisions}

Fpr the measurement of exclusive photoproduction of $\rho^{0}(770)$ mesons in the $\pi^{+} \pi^{-}$decay channel in ultra-peripheral $\mathrm{pPb}$ collisions at $\sqrt{s_{\mathrm{NN}}}=5.02 \mathrm{TeV}$ events were selected online by requiring the simultaneous presence of the two beams at the interaction point in conjunction with at least one track in the pixel tracker. Offline, events with the largest energy deposition in any of the HF (hadron forward) towers above the noise threshold of $3 \mathrm{GeV}$ are vetoed. Events are also required to have exactly two tracks corresponding to oppositely charged particles.

A large background is due to proton-dissociative events, $\gamma \mathrm{p} \rightarrow \rho^{0}(770) \mathrm{p}^{*}$, where $\mathrm{p}^{*}$ is a lowmass hadronic state. In order to determine this contribution, a sample of dissociative events is 

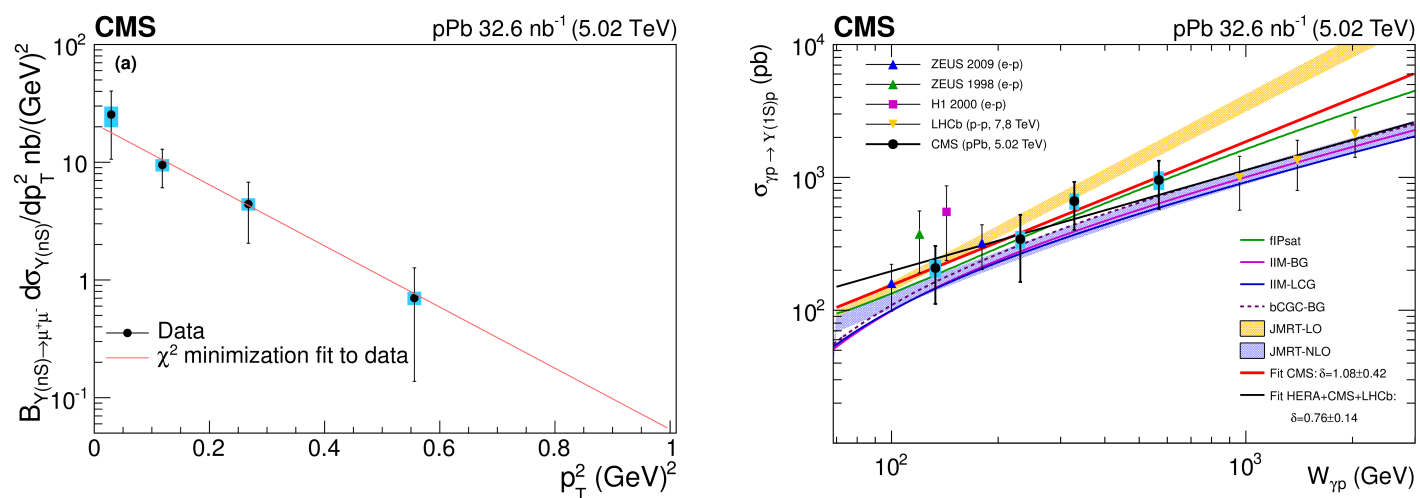

Figure 2: (Left) Differential $\Upsilon$ photoproduction cross section as a function of $|t|$ measured in $\mathrm{pPb}$ collisions at $\sqrt{s_{N N}}=5.02 \mathrm{TeV}$ in the dimuon rapidity region $|y|<2.2$. The solid line represents the result of a fit with an exponential function $N \cdot e^{b|t|}$. (Right) Cross section for exclusive $\Upsilon(1 S)$ photoproduction, $\gamma p \rightarrow \Upsilon(1 S) p$ as a function of photon-proton center-of-mass energy, $W_{\gamma p}[2]$.

selected by requiring activity in at least one of the forward detectors. This sample provides a template of the $p_{T}^{\pi^{+} \pi^{-}}$distribution of the dissociative events.

Another sizeable background comes from the exclusive photoproduction of $\rho(1700)$ mesons. The $\rho(1700)$ decays mostly into a $\rho^{0}(770)$ meson and a pion pair, leading to final states with four charged pions. It can mimic large $|t| \rho^{0}(770)$ events, when only two opposite-sign pions escape detection inducing a $p_{T}$ imbalance in the event and thus a distortion in the $|t|$ distribution. The template for this background is estimated using both data and MC samples.
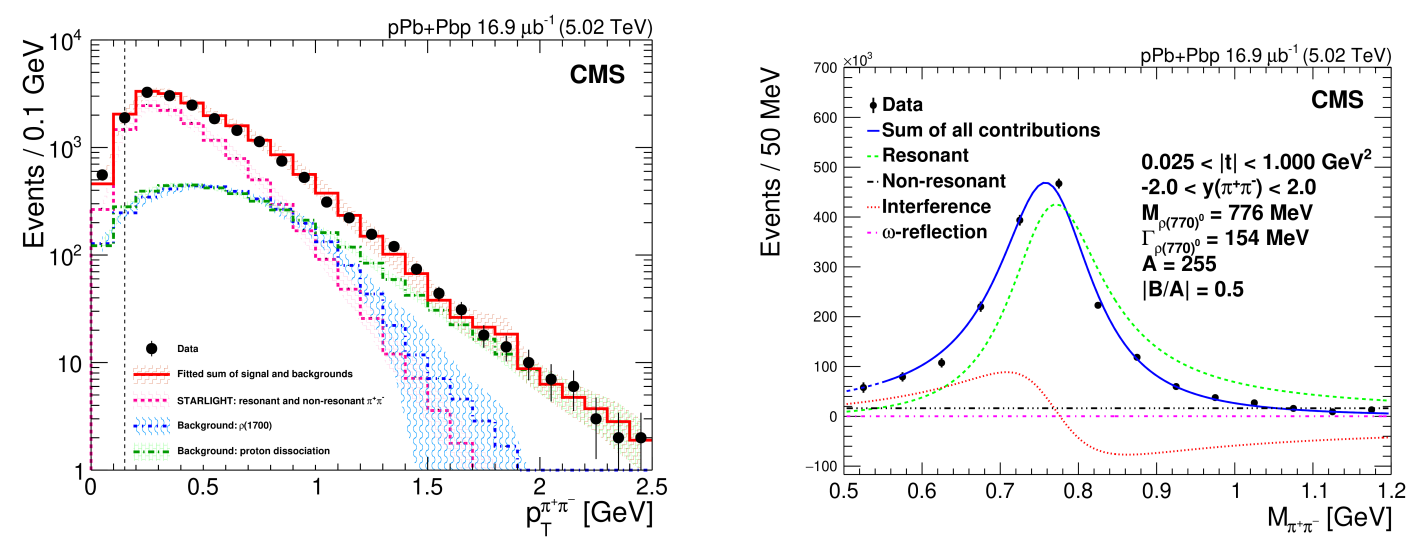

Figure 3: (Left) Template fit to the reconstructed $p_{T}$ of $\pi^{+} \pi^{-}$distribution. (Right) Invariant mass $M_{i n v}\left(\pi^{+} \pi^{-}\right)$distribution fitted with the Söding model 3.1.

The extraction of the signal contribution is carried out in two steps. First, the proton dissociative and the $\rho(1700)$ contributions are estimated by performing a fit to the data as a function of $p_{T}^{\pi^{+} \pi^{-}}$. This method relies on the fact that exclusive $\rho^{0}(770)$ events mainly contribute to the low$p_{T}^{\pi^{+} \pi^{-}}$region, non-exclusive events dominate the high $-p_{T}^{\pi^{+}} \pi^{-}$tail, while the $\rho(1700)$ contribution is at intermediate $p_{T}^{\pi^{+} \pi^{-}}$values. This makes the extraction of the proton dissociative and the $\rho(1700)$ contributions robust. The result of the fit of the $p_{T}^{\pi^{+} \pi^{-}}$distributions is shown in Fig. 3 (Left). The 
resulting residual proton-dissociative and $\rho(1700)$ contributions, over the whole rapidity interval, are $18 \pm 2 \%$ and $20 \pm 2 \%$, respectively.

After subtracting these backgrounds the invariant mass distribution is unfolded using the iterative D'Agostini method [12], which is regularized by four iterations. The invariant mass shape of the $\rho^{0}$ in photoproduction is then fitted to the Söding formula [13]:

$$
\frac{\mathrm{d} N_{\pi^{+} \pi^{-}}}{\mathrm{d} M_{\pi^{+} \pi^{-}}}=\left|A \frac{\sqrt{M_{\pi^{+} \pi^{-}} M_{\rho} \Gamma\left(M_{\pi^{+} \pi^{-}}\right)}}{M_{\pi^{+} \pi^{-}}^{2}-M_{\rho^{0}}^{2}+i M_{\rho^{0}} \Gamma\left(M_{\pi^{+} \pi^{-}}\right)}+B\right|^{2},
$$

where $A$ is the amplitude of the Breit-Wigner function and $B$ is the amplitude of the direct nonresonant $\pi^{+} \pi^{-}$production.

Figure 3 (Right) shows the fit of the unfolded distribution, which gives $M_{\rho^{0}}=776 \pm 1$ (stat) $\mathrm{MeV}$ and $\Gamma_{\rho^{0}}=154 \pm 3$ (stat) MeV, consistent with the world average values [14]. The ratio of the nonresonant to resonant contributions, $|B / A|$, is $0.50 \pm 0.05$ (stat), lower than $0.57 \pm 0.09$ (stat) and $0.70 \pm 0.04$ (stat), the values reported by the H1 and ZEUS Collaborations, respectively $[15,16]$. In addition, no significant dependence of this ratio on $W_{\gamma p}$ is found, in agreement with results reported by ZEUS.

The differential cross section for exclusive photoproduction is given by

$$
\frac{\mathrm{d} \sigma}{\mathrm{d} y}=\frac{N_{\rho^{0}}^{\mathrm{exc}}}{\mathscr{B} L \Delta y},
$$

where $N_{\rho^{0}}^{\text {exc }}$ is the corrected number of exclusive $\rho^{0}$ events obtained from the fits; $\mathscr{B}$ is the branching fraction, equal about 0.99 [14], $\Delta y$ is the rapidity interval, and $L$ is the integrated luminosity of the data sample. The cross section $\frac{\mathrm{d} \sigma}{\mathrm{d} y}\left(\mathrm{pPb} \rightarrow \mathrm{pPb} \rho^{0}\right)$ is related to the photon-proton cross section, $\sigma\left(\gamma \mathrm{p} \rightarrow \rho^{0} \mathrm{p}\right) \equiv \sigma\left(W_{\gamma \mathrm{p}}\right)$, through the photon flux, $\frac{\mathrm{d} n}{\mathrm{~d} k}$ :

$$
\frac{\mathrm{d} \sigma}{\mathrm{d} y}\left(\mathrm{pPb} \rightarrow \mathrm{pPb} \rho^{0}\right)=k \frac{\mathrm{d} n}{\mathrm{~d} k} \sigma\left(\gamma \mathrm{p} \rightarrow \rho^{0} \mathrm{p}\right) .
$$

Here, $k$ is the photon energy, which is determined from the $\rho^{0}$ mass and rapidity, according to the formula $k=(1 / 2) M_{\rho^{0}} \exp \left(-y_{\rho^{0}}\right)$. The average photon flux and the average centre-of-mass energy $\left(\left\langle W_{\gamma p}\right\rangle\right)$ values in each rapidity interval are calculated using STARLIGHT. The resulting photonproton cross section, obtained for $W_{\gamma_{\mathrm{p}}}$ between 29 and $213 \mathrm{GeV}\left(\left\langle W_{\gamma_{\mathrm{p}}}\right\rangle=92.6 \mathrm{GeV}\right)$ and integrated over the range $0<|t|<0.5 \mathrm{GeV}^{2}$, is $\sigma=11.2 \pm 1.4$ (stat) \pm 1.0 (syst) $\mu \mathrm{b}$. The photon-proton cross section values, $\sigma\left(\gamma \mathrm{p} \rightarrow \rho^{0} \mathrm{p}\right)$, for all rapidity bins are presented in Fig. 4 . Figure 4 (left) also shows a compilation of the fixed-target [17] and HERA results [15, 16]. The line indicates the result of a fit to all the plotted data with the formula $\sigma=\alpha_{1} W_{\gamma_{\mathrm{p}}}^{\delta_{1}}+\alpha_{2} W_{\gamma_{\mathrm{p}}}^{\delta_{2}}$. The fit describes the data well. The CMS and HERA data are also fitted with the function $\sigma=\alpha W_{\gamma \mathrm{p}}^{\delta}$. The fit yields $\delta=0.23 \pm$ 0.14 (stat) \pm 0.04 (syst).

The unfolded invariant mass distribution is studied for different $|t|$ bins, and the extraction of the $\rho^{0}$ photoproduction cross section is repeated in each bin. In order to compare with the HERA results, the $p_{T}$-related measurements are presented in terms of $|t|$, approximated as $|t| \approx\left(p_{T}^{\pi^{+} \pi^{-}}\right)^{2}$.

Figure 4 (right) shows the differential cross section $\mathrm{d} \sigma / \mathrm{d}|\mathrm{t}|$ in the rapidity interval $-1.2<$ $y\left(\pi^{+} \pi^{-}\right)<0$, corresponding to a $W_{\gamma p}$ range similar to that at HERA, compared with the H1 and 

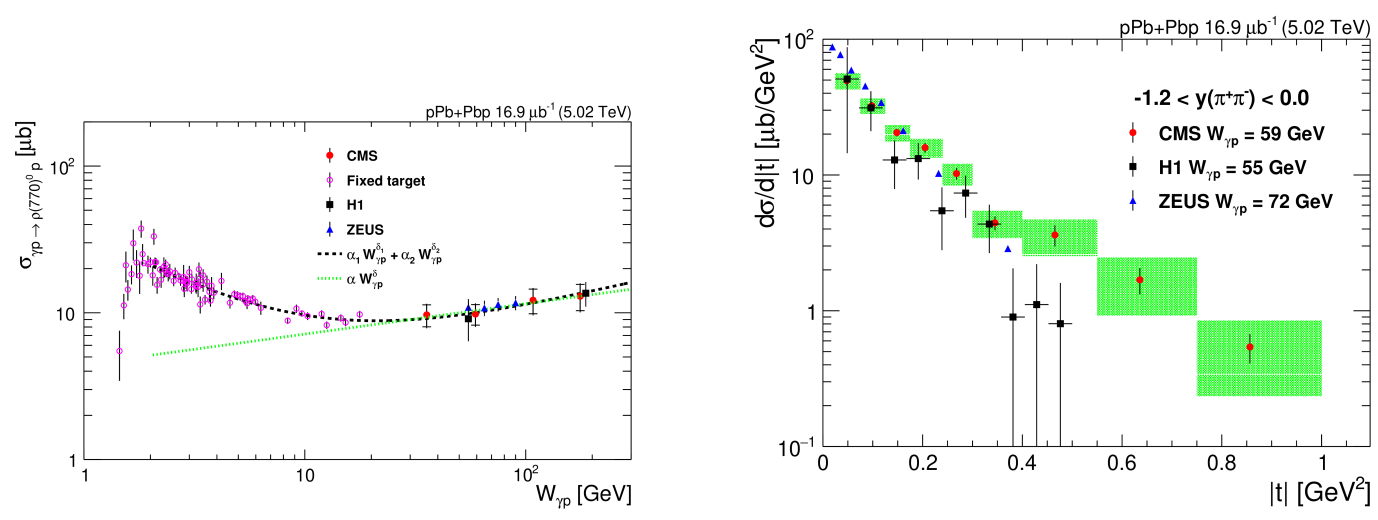

Figure 4: (Left) The total cross section $\sigma$ of $\rho^{0}$ photoproduction [18]. (Right) Differential cross section $\mathrm{d} \sigma / \mathrm{d}|\mathrm{t}|$ for exclusive $\rho^{0}$ photoproduction [18] together with H1 and ZEUS data [15, 16]

ZEUS results $[15,16]$. The Regge formula [19] $b=b_{0}+2 \alpha^{\prime} \cdot \ln \left(\frac{W_{\gamma p}}{W_{0}}\right)^{2}$, which parametrizes the dependence of the exponential slope $b$ of the differential cross section on the collisions energy. The fit to the CMS data alone gives a pomeron slope $\alpha^{\prime}=0.48 \pm 0.33$ (stat) \pm 0.12 (syst) $\mathrm{GeV}^{-2}$, consistent with the ZEUS [16] value and the Regge expectation of $0.25 \mathrm{GeV}^{-2}$.

\section{References}

[1] S. Chatrchyan et al. [CMS Collaboration], JINST 3 (2008) S08004.

[2] A. M. Sirunyan et al. [CMS Collaboration], Eur. Phys. J. C 79 (2019) no.3, 277

[3] R. Aaij et al. [LHCb Collaboration], JHEP 1509 (2015) 084

[4] H. Abramowicz et al. [ZEUS Collaboration], Phys. Lett. B 708 (2012) 14

[5] S. P. Jones, A. D. Martin, M. G. Ryskin and T. Teubner, JHEP 1311 (2013) 085

[6] A. Aktas et al. [H1 Collaboration], Eur. Phys. J. C 46 (2006) 585

[7] S. Chekanov et al. [ZEUS Collaboration], Phys. Lett. B 680 (2009) 4

[8] T. Lappi and H. Mantysaari, Phys. Rev. C 83 (2011) 065202

[9] G. S. d. Santos and M. V. T. Machado, Phys. Rev. C 89 (2014) no.2, 025201

[10] G. Sampaio dos Santos and M. V. T. Machado, J. Phys. G 42 (2015) no.10, 105001

[11] V. P. Goncalves, B. D. Moreira and F. S. Navarra, Phys. Lett. B 742 (2015) 172

[12] G. D’ Agostini, Nucl. Instrum. Meth. A 362 (1995) 487

[13] P. Soding, Phys. Lett. 19 (1966) 702

[14] C. Patrignani et al. [Particle Data Group], Chin. Phys. C 40 (2016) no.10, 100001

[15] S. Aid et al. [H1 Collaboration], Nucl. Phys. B 463 (1996) 3

[16] J. Breitweg et al. [ZEUS Collaboration], Eur. Phys. J. C 2 (1998) 247

[17] S. A. Morrow et al. [CLAS Collaboration], Eur. Phys. J. A 39 (2009) 5

[18] A. M. Sirunyan et al. [CMS Collaboration], arXiv:1902.01339 [hep-ex].

[19] K. A. Goulianos, Phys. Rept. 101 (1983) 169 